\title{
e-MERLIN Algorithm Development
}

\section{Fenech, ${ }^{1}$ I. Stewart ${ }^{2}$ and S. Garrington ${ }^{* 2}$}

${ }^{1}$ Department of Physics and Astronomy, University College London

2 Jodrell Bank Centre for Astrophysics, University of Manchester

E-mail: dmfestar.ucl.ac.uk

e-MERLIN is a major upgrade to the MERLIN array which consists of seven linked radio telescopes situated across the UK, including the Lovell telescope at Jodrell Bank Observatory. In order to make use of the $\sim 30$-fold increase in sensitivity that this upgrade will provide, as well as the wide-band and wide-field capabilities, development of the data reduction algorithms and procedures are essential. We present an overview of the implementation of the Sault-Wieringa wide-band imaging algorithm that will be used for e-MERLIN imaging as well as the progress of testing this algorithm on multi-frequency MERLIN observations of the nearby starburst galaxy, M82.

The 9th European VLBI Network Symposium on The role of VLBI in the Golden Age for Radio Astronomy and EVN Users Meeting

September 23-26, 2008

Bologna, Italy

\footnotetext{
* Speaker.
} 


\section{Introduction}

The upgrade to the MERLIN (Multi-Element Radio Linked Interferometry Network) array based at Jodrell Bank Observatory includes new receivers and the replacing of the existing microwave links with fibre-optic cable. This will give rise to the new instrument e-MERLIN, an array that will operate at three frequency bands: L $(1.2-1.4 \mathrm{GHz}), \mathrm{C}(4-8 \mathrm{GHz})$ and $\mathrm{K}(22-24 \mathrm{GHz})$ with $2 \mathrm{GHz}$ bandwidth. The upgrade in total will provide an almost complete aperture coverage at $\mathrm{C}$ band and result in an $\sim 30$-fold increase in sensitivity, achieving $\mu$ Jy noise levels in around $12 \mathrm{hrs}$. The use of such large bandwidths requires the implementation of new algorithms to correct for the varying brightness of the radio sources of interest as a function of frequency.

\section{Wide-band imaging}

The majority of the observed radiation from radio sources is usually either synchrotron or thermal continuum emission, which varies as a function of frequency and can be described by a power-law function defined by the spectral index, $\alpha$. The use of large bandwidths as for eMERLIN, will produce spectral artifacts when imaging such radio sources, unless these frequencydependent variations are corrected for.

In a standard interferometric observation, the array samples the visibility function,

$$
V(u, v)=S(u, v) * I(u, v)
$$

Taking the Fourier transform of this function gives rise to the dirty image

$$
I^{\prime}(x, y)=I(x, y) * B_{0}(x, y)
$$

where $I$ is the true source distribution and $B_{0}$ is the dirty beam.

However, when using large bandwidths it is necessary to introduce a spectral variation into the source brightness distribution, thus $I(x, y)$ becomes $I\left(x, y, v_{0}\right)\left(v / v_{0}\right)^{-\alpha}$. It is now possible to expand the expression for the dirty image using (though this is not restricted to) a Taylor expansion, as described by $[1-3]$. This provides the final expression for the dirty image,

$$
I^{\prime}=\left(I * B_{0}\right)+\left(I \alpha * B_{1}\right)+1 / 2\left[I \alpha(\alpha+1) * B_{2}\right] \ldots \ldots
$$

where $I^{\prime}$ is a function of the frequency, $v$ and spectral index, $\alpha$ (written here up to the second order of the Taylor expansion).

Hence, when performing the cleaning process during imaging, the effects of the 'normal' dirty beam, $B_{0}$ as well as the spectral dirty beams, $B_{1}$ and $B_{2}$ have to be removed from the dirty image. Figure 1 shows an example of the form of the normal and spectral dirty beams for e-MERLIN.

For e-MERLIN, this process will be performed using a Python/Parseltongue implementation of the Sault-Wieringa algorithm, which will clean the dirty beams from the dirty image in parallel, see [3] for a full explanation of this algorithm. 


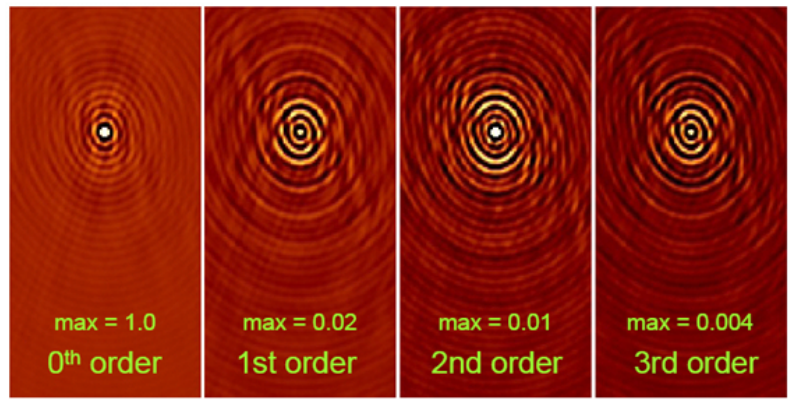

Figure 1: An example of the normal and spectral dirty beams used for the Sault-Wieringa wide-band algorithm for imaging e-MERLIN observations.

\section{Testing the wide-band algorithm}

In order to test the implementation of the Sault-Wieringa algorithm prior to being able to observe with e-MERLIN, simulations as well as a collection of observations spaced in frequency made with MERLIN will be used. Figure 2 shows a comparison of the use of standard and wideband cleaning of an image containing a collection of point sources of varying size, brightness and spectral index formed from simulated observations with $2 \mathrm{GHz}$ bandwidth.
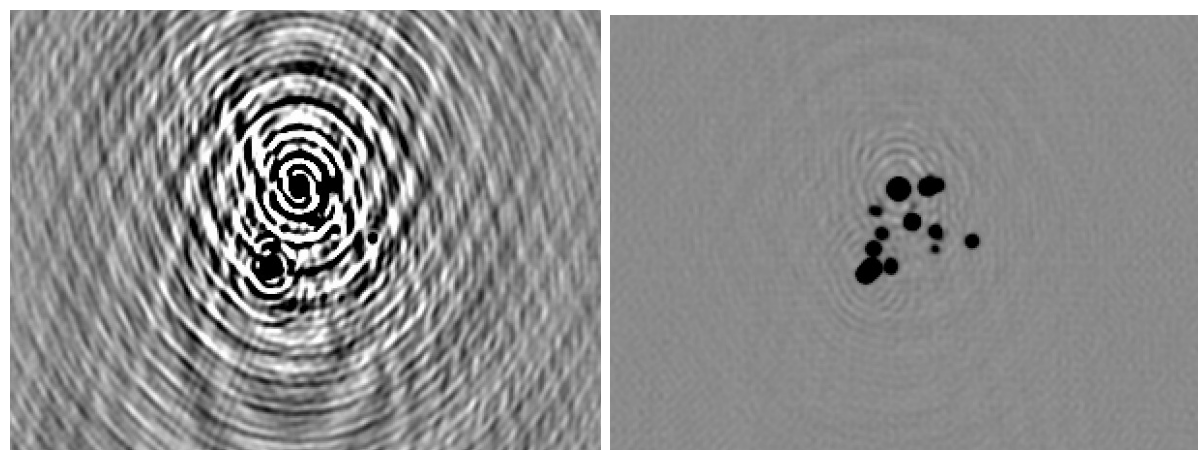

Figure 2: A comparison of the Högbom and (second order) Sault-Wieringa cleaning of an image of point sources with varying spectral index.

The MERLIN observations that will also be used are of the nearby $(\sim 3.2 \mathrm{Mpc})$ starburst galaxy, M82. This galaxy is well studied [4-7] and is known to contain around 50 discrete sources, both supernova remnants (SNR) and HII regions, providing an ideal test for the wide-band algorithm.

The observations were made between Oct. 2006 and Jun. 2007 at ten separate frequencies between 4.5 and $6.7 \mathrm{GHz}$. All of the observations were made with $16 \mathrm{MHz}$ bandwidth, split into 32 channels. Each dataset has been calibrated separately using $3 \mathrm{C} 286$ to set the amplitude scale and 0955+697 to calibrate the telescope phases. In order to fully test the algorithm, it is necessary to combine the data in the $\mathrm{u}, \mathrm{v}$-plane to form a single large dataset representative of the format of a standard e-MERLIN dataset, which can then be gridded and processed using the Taylor expansion dirty beams. However, for the purposes of initially processing a small number of the M82 datasets 

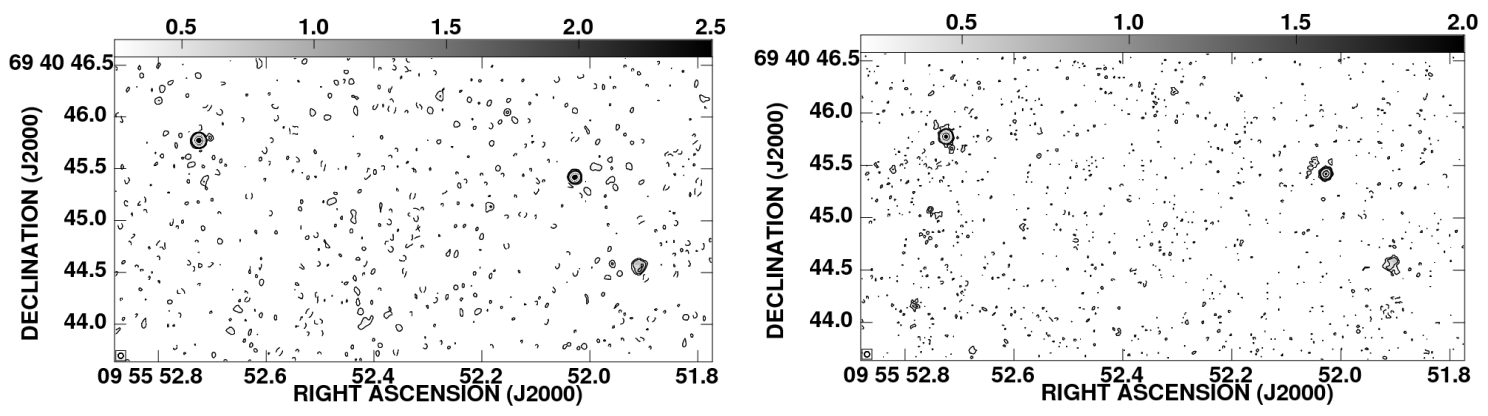

Figure 3: A sub-image of M82 showing three supernova remnants in the central region of M82 at 4546 and $6670 \mathrm{MHz}$.

together, it is possible to make individual dirty images at each frequency and combine them to form the required dirty image. The dirty beams used in this case are then just the individual frequency dirty beams.

The first datasets to be processed in this way are those at 4546, 6183.6 and $6670 \mathrm{MHz}$ and Figure 3 shows the same sub-image from two of these datasets. Figure 4 shows a resulting RGB image of the supernova remnant (SNR) 40.68+55.1 from the imaging and cleaning of these data using the wide-band techniques described.
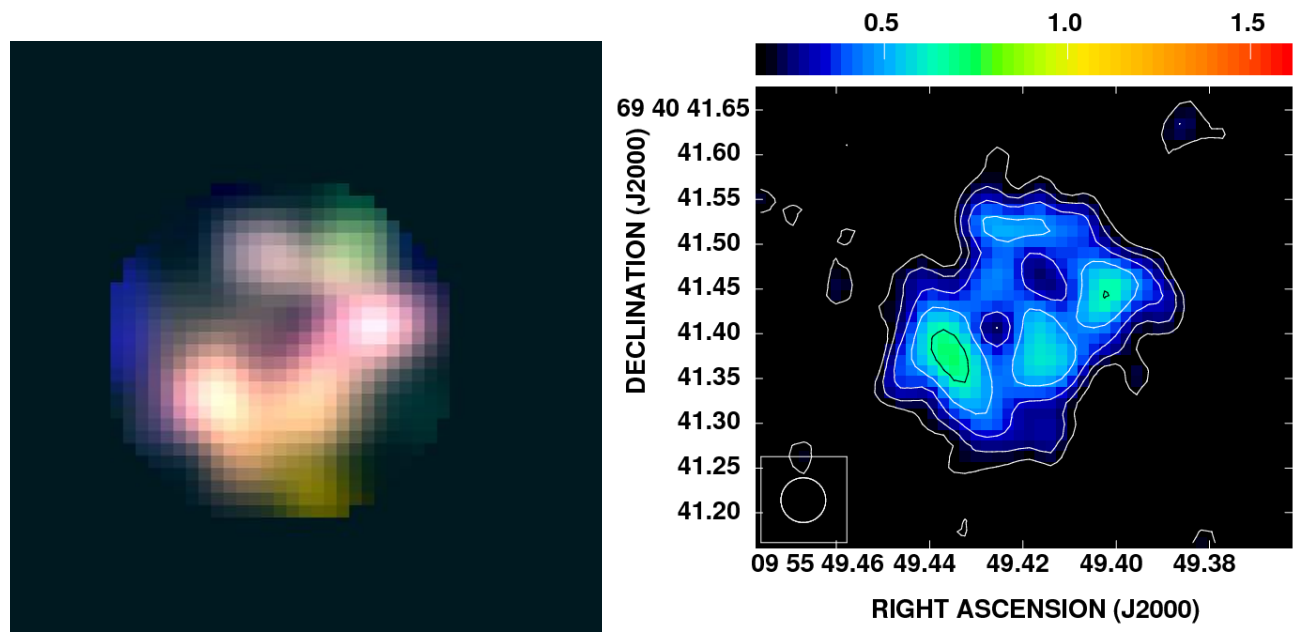

Figure 4: Left: A three-colour image of the the SNR 40.68+55.1, processed using the Sault-Wieringa algorithm. The three colours show the contributions of the three frequencies, 4546, 6183.6 and $6670 \mathrm{MHz}$. Right: Three frequencies of M82 data combined and processed through IMAGR using a spectral index correction of -0.7 . Both images are restored with a 50 mas circular beam.

\section{4. e-MERLIN data processing}

Many of the current data-reduction procedures used for calibration and imaging require some adaptation for use with the large datasets and wide-band capabilities of the next generation of radio 
telescopes. In particular large sections of this process will necessitate pipelining in order to make calibration and use of e-MERLIN data accessible to non-radio astronomers. Figure 5 shows the current basic data-flow outline for e-MERLIN, the majority of which is expected to be pipelined using Python/Parseltongue for ease of interaction with the current data processing software such as AIPS and CASA.

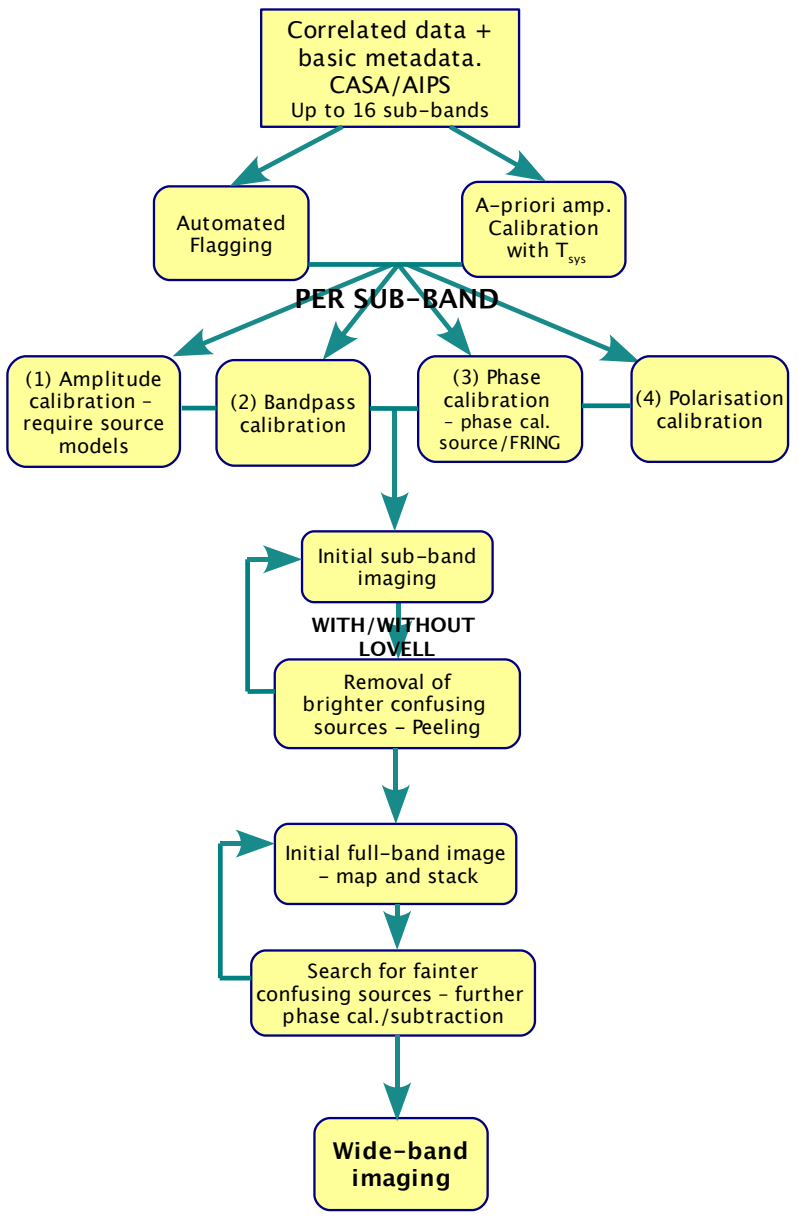

Figure 5: An outline of the proposed stages of data reduction and imaging for e-MERLIN observations.

\section{Conclusions}

An overview of the current developments of wide-band imaging for the e-MERLIN array and the successful test of the Sault-Wieringa algorithm using multi-frequency MERLIN observations of the starburst galaxy, M82 have been presented. The implementation of wide-band, wide-field 
imaging algorithms will be essential to the success of the next generation of radio telescopes such as e-MERLIN, ELVA and ALMA which will make use of large instantaneous bandwidths.

\section{Acknowledgments}

MERLIN is a national facility operated by the University of Manchester on behalf of the Science and Technologies Facilities Council (STFC). PARSELTONGUE was developed in the context of the ALBUS project, which has benefited from research funding from the European Community's sixth Framework Programme under RadioNet R113CT 20035058187.

\section{References}

[1] J. E. Conway, T. J. Cornwell and P. N. Wilkinson, Multi-frequency synthesis: a new technique in radio interferometric imaging, MNRAS 1990 (246) 490

[2] J. E. Conway and R.J. Sault, Multi-frequency synthesis, in proceedings of Very Long Baseline Interferometry and the VLBA, ASP Conference Series, 1995 (82) 310

[3] R. J. Sault and M. H. Wieringa, Multi-frequency synthesis techniques in radio interferometric imaging, Astron. Astrophys. Suppl. Ser., 1994 (108) 585

[4] T.W.B. Muxlow, A. Pedlar, P.N. Wilkinson, D.J. Axon, E.M. Sanders, A.G. deBruyn, The Structure of Young Supernova Remnants in M82, MNRAS 1994 (266) 455

[5] K.A. Wills, A. Pedlar, T.W.B. Muxlow, P.N. Wilkinson, Low-frequency observations of supernova remnants in M82, MNRAS 1997 (291) 517

[6] A. R. McDonald, T. W. B. Muxlow, K. A. Wills, A. Pedlar and R. J. Beswick, A parsec-scale study of the 5/15-GHz spectral indices of the compact radio sources in M82, MNRAS, 2002 (334) 912

[7] D. M. Fenech, T. W. B. Muxlow, R. J. Beswick, A. Pedlar and M. K. Argo, Deep MERLIN 5GHz radio imaging of supernova remnants in the M82 starburst MNRAS, 2008 (391) 1384 